Rev. Bras. Saúde Prod. Anim., Salvador, v.15, n.2, p.425-429 abr./jun., 2014 http://www.rbspa.ufba.br ISSN 15199940

\title{
Uso do acetato de melengestrol após protocolos de inseminação artificial em tempo fixo em vacas nelore multíparas
}

\author{
Use of melengestrolacetate after use protocol to artificial insemination at fixed-time in \\ Nellore cows pluriparous
}

\author{
SILVA JUNIOR, Lourival de Souza e ${ }^{1}$; FREIRIA, Lucien Bissi da ${ }^{2 *}$; ANGREVES- \\ SILVA, Giselde Marques ${ }^{3}$; POSSAMAI, Adriano Jorge ${ }^{2}$; HATAMOTO- \\ ZERVOUDAKIS, Luciana Keiko ${ }^{2}$; SILVA, Mérik Rocha ${ }^{2}$
}

\footnotetext{
${ }^{1}$ Instituto de Defesa Agropecuária, Fiscal de Defesa Agropecuário, Pontes e Lacerda, Mato Grosso, Brasil.

${ }^{2}$ Universidade Federal do Mato Grosso, Faculdade de Agronomia, Medicina Veterinária e Zootecnia, Programa de Pós-Graduação em Ciência Animal, Cuiabá, Mato Grosso, Brasil.

${ }^{3}$ Universidade do Estado do Mato Grosso, Departamento de Zootecnia, Pontes e Lacerda, Mato Grosso, Brasil.

*Endereço para correspondência: lucienbissi@hotmail.com
}

\section{RESUMO}

Objetivou-se avaliar a influência do fornecimento de 2,28g de $\mathrm{MGA}^{\circledR}$ Premix entre o $13^{\circ}$ e $17^{\circ}$ dia após a inseminação artificial em tempo fixo (IATF), na taxa de gestação de vacas da raça Nelore, pluríparas. Foram usadas 154 vacas, divididas em dois lotes ao acaso, o tratamento $1, n=55$ e o tratamento $2, n=99$. O tratamento 1 recebeu suplemento mineral e o tratamento 20 mesmo suplemento adicionado de 2,28g de $\mathrm{MGA}^{\circledR}$ Premix/animal/dia. Foi usada a estatística descritiva por tabela frequência, no programa SAS, pela interação do fornecimento de MGA $^{\circledR}$ Premix com a taxa de gestação. A porcentagem de gestação foi superior para as vacas suplementadas com MGA $^{\circledR}$ Premix e avaliados aos 43 dias após a inseminação. $\mathrm{O}$ uso de MGA $^{\circledR}$ após IATF aumenta a sobrevivência embrionária e a taxa de prenhez.

Palavras-chave: escore de condição corporal, ordem de parto, progestágeno, sincronização do estro, zebuíno

\section{SUMMARY}

Objective was to evaluate the effects of supply $2.28 \mathrm{~g} \mathrm{MGA}^{\circledR}$ Premix between the 13th and 17th day after fixed-time artificial insemination (TFAI), on pregnancy rate of multiparous Nellore cows. It used 154 cows, divided randomly into two lots, Treatments $1, \mathrm{n}=55$ and Treatments $2, \mathrm{n}=99$. The treatments 1 received only one mineral supplement and treatments 2 received the same batch mineral supplement added 2.28g $\mathrm{MGA}^{\circledR}$ Premix/animal/day. We used descriptive statistics for frequency table, on program SAS, by the interaction of supply $\mathrm{MGA}^{\circledR}$ Premix with the rate of pregnancy. The pregnancy rate was higher for cows supplemented MGA ${ }^{\circledR}$ Premix evaluated at 43 days of pregnancy. Using TFAI after $M_{G A}{ }^{\circledR}$ Premix increases the rate of embryo survival and rate of pregnancy.

Keywords: body condition score, progestin, order of birth, synchronization of estrus, zebu

\section{INTRODUÇÃO}

A inseminação artificial (IA) é uma das mais difundidas biotecnologias utilizadas como instrumento eficaz e econômico na reprodução de bovinos (NEVES et al., 2010). No entanto, seu uso vem se restringindo em função dos desafios operacionais, em particular quanto ao longo período de observação das matrizes e falhas no registro de estro clínico. Isto impacta fortemente a eficiência reprodutiva do rebanho, mormente no 
Rev. Bras. Saúde Prod. Anim., Salvador, v.15, n.2, p.425-429 abr./jun., 2014 http://www.rbspa.ufba.br ISSN 15199940

tocante a duração do intervalo entre partos, o que leva a redução no número de crias nascidas por ano (GOFERT, 2008).

Diante destes entraves, foram desenvolvidos protocolos de inseminação artificial em tempo fixo (IATF), o que dispensa a necessidade de observação das matrizes para a ocorrência de estro (GOFERT, 2008) Estes protocolos lançam mão da sincronização do estro e da ovulação e permitem obter gestação em torno de $50,0 \%$ após a primeira inseminação (NEVES et al., 2010). Possivelmente, o principal fator limitante na utilização destes protocolos está no reduzido reconhecimento da gestação e consequente implantação do embrião. Evidencie-se que a falha do reconhecimento materno da prenhez provocada pela insuficiência do corpo lúteo em sintetizar e secretar níveis adequados de progesterona favorece a mortalidade precoce do embrião (MACHADO et al., 2010). Desta forma, a interação entre o ambiente uterino e o embrião é essencial para garantir a sobrevivência deste, sendo a progesterona um importante indutor das mudanças uterinas, além da sua ação estar correlacionada com o crescimento embrionário (CARTER et al., 2008). Assim, entende-se que a administração de progesterona pós-inseminação artificial possivelmente melhora o desenvolvimento do embrião, aumentando as taxas de concepção e sobrevivência (AONO et al., 2008).

$\mathrm{O}$ acetato de melengestrol MGA ${ }^{\circledR}$ é um progestágeno de administração oral. $\mathrm{O}$ seu uso é recomendado para melhorias na fixação e taxas de concepção de vacas em anestro pós-parto, face auxiliar na sincronia do estro e indução da ovulação (WOOD-FOLLIS et al., 2004). Com este enfoque objetivou-se avaliar a influência do fornecimento, por via oral, de $2,28 \mathrm{~g}$ de $\mathrm{MGA}^{\circledR}$ do $13^{\circ}$ ao $17^{\circ}$ dia após IATF na taxa de gestação de vacas Nelore, multíparas.

\section{MATERIAIS E MÉTODOS}

O experimento foi conduzido na Fazenda do Sagrado Coração de Jesus em Vila Bela da Santíssima Trindade, Mato Grosso, no período de Abril a Maio de 2011. Foram usadas 154 vacas, pluríparas, da raça Nelore, puras de origem (PO), com partos ocorridos no mês de fevereiro de 2011, aos 60 dias pós-parto. Todos os animais foram submetidos à avaliação do escore de condição corporal (ECC), por um único técnico, usando a escala de 1 a 5 pontos (Meneghetti \& Vasconcelos, 2008) e todas as matrizes incluídas no experimento estavam com o ECC corporal 3.

As vacas foram divididas, ao acaso, em dois tratamentos. O tratamento $1 \mathrm{com}$ 55 vacas e o tratamento 2 com 99 vacas.

$\mathrm{O}$ tratamento 1 recebeu apenas suplemento mineral (SM) ad libitum (Tabela 1) e o tratamento 2, o mesmo suplemento com adição de $2,28 \mathrm{~g}$ de MGA ${ }^{\circledR}$ Premix/animal/dia, até o $17^{\circ}$ dia, restringindo-se a disponibilidade do suplemento mineral-MGA em $70 \mathrm{~g} / \mathrm{UA} / \mathrm{dia}$.

As vacas, independente de tratamento foram mantidos em piquetes formados uniformemente por Brachiaria Brizantha cv. Marandu e tinham acesso livre à água. O saleiro foi instalado a um (1) metro de altura do solo para evitar o consumo do suplemento pelas crias, e permitia acesso simultâneo das matrizes.

A realização da IATF seguiu o protocolo comercial (Figura 1) e a partir do $13^{\circ}$ dia da IA iniciou-se o fornecimento do suplemento, enriquecido ou não, com 2,28g de $\mathrm{MGA}^{\circledR}$ Premix por animal/dia. A avaliação da eficiência do suplemento 
Rev. Bras. Saúde Prod. Anim., Salvador, v.15, n.2, p.425-429 abr./jun., 2014 http://www.rbspa.ufba.br ISSN 15199940

usado após a IATF sobre o reconhecimento materno da gestação foi feita por meio da confirmação da prenhez por ultrassonografia no D 43, isto é, 30 dias após o início da disponibilidade do suplemento, usandose o equipamento MINDRY DP-2200 VET, com transdutor linear de 7,5 Mhz.
Para análise dos dados, foi utilizada uma estatística descritiva por tabela de frequência, com nível de significância de $10 \%$ pelo teste de qui-quadrado. Os dados foram analisados pelo programa SAS, pela interação dos tratamentos com a taxa de gestação.

Tabela 1. Níveis de garantia por kg do suplemento mineral

\begin{tabular}{lc}
\hline Composição & Quantidade $(\mathrm{g} / \mathrm{kg})$ \\
\hline Cálcio (máx.) & 200,000 \\
Cálcio (mín.) & 175,000 \\
Fósforo (mín.) & 87,000 \\
Sódio (mín.) & 120,000 \\
Magnésio (mín.) & 10,000 \\
Enxofre (mín.) & 14,000 \\
Zinco (mín.) & 5,500 \\
Cobre (mín.) & 1,600 \\
Manganês (mín.) & 1,300 \\
Cobalto (mín.) & 0,072 \\
Selênio (mín.) & 0,030 \\
Iodo (mín.) & 0,072 \\
Flúor (máx.) & 0,870 \\
Umidade (máx.) & 120,000 \\
\hline
\end{tabular}

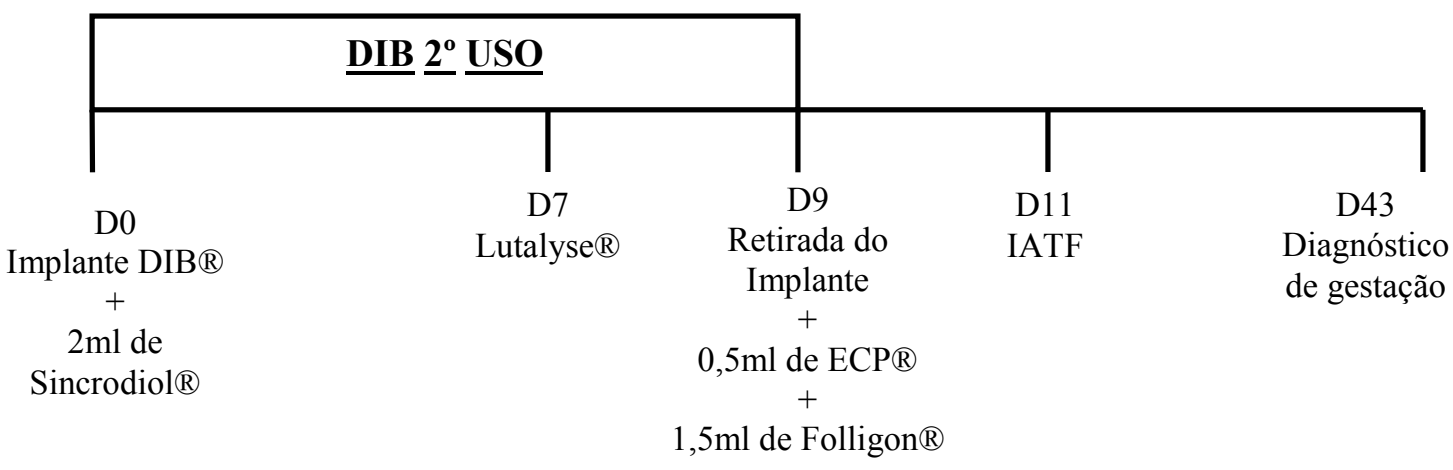

Figura 1. Protocolo experimental

\section{RESULTADOS E DISCUSSÃO}

O uso de $2,28 \mathrm{~g}$ de $\mathrm{MGA}^{\circledR}$ Premix/animal/dia promoveu maior taxa de prenhez $(\mathrm{P}<0,01)$, conforme descrito na Tabela 2.
Os resultados do presente trabalho condizem com aqueles descritos por Aono et al. (2008), ao avaliaram o efeito do fornecimento do MGA, por sete dias $\left(14^{\circ}\right.$ ao $\left.20^{\circ}\right)$ após IATF associado à remoção temporária da cria para sincronizar o estro de vacas Nelore 
Rev. Bras. Saúde Prod. Anim., Salvador, v.15, n.2, p.425-429 abr./jun., 2014 http://www.rbspa.ufba.br ISSN 15199940

com cria ao pé após serem submetidas à IATF, verificaram-se que os animais que receberam $\mathrm{MGA}^{\circledR}$ em relação aos não tratados, tiveram maior taxa de prenhez à IATF $(51,8 \%$ e $32,8 \%$, respectivamente) e maior taxa de retorno ao cio $(41,5 \%$ e $26,0 \%$, respectivamente).

Tabela 2. Frequência estatística de vacas gestantes e não gestantes nos tratamentos 1 e 2

\begin{tabular}{cccc}
\hline Tratamento & $\mathrm{N}^{\mathrm{o}}$ de animais & Prenhez positiva & Prenhez negativa \\
\hline 1 & 55 & $18,00^{\mathrm{A}}$ & 17,33 \\
2 & 99 & $48,67^{\mathrm{B}}$ & 16,00 \\
\hline
\end{tabular}

Médias seguidas de letras diferentes na mesma coluna diferem pelo teste qui-quadrado $(\mathrm{P}<0,01)$.

Garret et al. (1988), avaliaram a influência da administração de $100 \mathrm{mg}$ de progesterona exógena nos quatro (4) primeiros dias de gestação sobre 0 desenvolvimento e o crescimento do concepto bovino e concluíram que os animais tratados apresentaram maiores concentrações plasmática de progesterona no início da gestação e mais proteína no líquido uterino aos 14 dias de gestação, além de os embriões terem apresentado um melhor desenvolvimento.

De acordo com Mann \& Lamming (1999), quando é realizada a suplementação exógena de progesterona, ocorrem à diminuição das perdas embrionárias de, em média, $5,0 \%$. E, se a suplementação for feita antes do $6^{\circ}$ dia de gestação os ganhos podem chegar até a $10,0 \%$ a mais de prenhez. Ainda, quando o rebanho tem o histórico de não mais do que 50,0\% de gestação, a eficiência da suplementação pode propiciar o incremento de até $19,0 \%$.

Robison et al. (1989), ao fazerem a suplementação de progesterona por meio do dispositivo intravaginal (PRID) no início da gestação, obteve prenhez maior nos animais tratados $(60,0 \%)$ do que naqueles não tratados $(30,0 \%)$. $O$ que corrobora com os resultados aqui apresentados.
Sá Filho e Vasconcelos (2010) obtiveram taxa de concepção de 50,6\% ao usarem 316 matrizes no pós-parto de até 110 dias, após uma única IATF. Porcentagem semelhante à descrita por Ereno et al. (2007), ao usarem 87 vacas Nelore com até 90 dias pós-parto. Portanto, o fornecimento de progestágeno promove aumento considerável na taxa de concepção em uma única IATF. Desta forma o seu emprego após protocolos comerciais é capaz de aumentar a eficiência reprodutiva do rebanho.

Conclui-se que o uso de suplemento mineral enriquecido com $\mathrm{MGA}^{\circledR}$ após a IATF aumenta a taxa de concepção em vacas da raça Nelore.

\section{REFERÊNCIAS}

AONO, F.H.S; PERES, R.F.G.; MARCON, C.C. Utilização de MGA premix associado á remoção temporária do bezerro para sincronizar o cio de retorno após IATF em vacas nelore paridas. Acta Scientiae Veterinariae, v.36, p.622, 2008. 
Rev. Bras. Saúde Prod. Anim., Salvador, v.15, n.2, p.425-429 abr./jun., 2014 http://www.rbspa.ufba.br ISSN 15199940

CARTER, F.; FORDE, N.; DUFFY, P.; WADE, M.; FAIR, T.; CROWE, M.A.; EVANS, A.C.O.; KENNY, D.A.;

ROCHE, J.F.; LONERGAN, P. Effect

of increasing progesterone

concentration from Day 3 of pregnancy on subsequent embryo survival and development in beef heifers.

Reproduction, Fertility and

Development, v.20, n.3, p.368-375, 2008.

ERENO, R.L.; BARREIROS, T.R.R.; SENEDA, M. M.; BARUSELLI, P.S.; PEGORER, M.F.; BARROS, C.M. Taxa de prenhez de vacas Nelore lactantes tratadas com progesterona associada à remoção temporária de bezerros ou aplicação de gonadotrofina coriônica eqüina. Revista Brasileira de Zootecnia, v.36, n.5, p.1288-1294, 2007.

GARRET, J.E.; GEISERT, J.E.; ZAVY, M.T.; MORGAN, G.L.Evidence for maternal regulation of early conceptus growth and development in beef cattle.

Journal of Reproduction and Fertility, v.84, p.437-446, 1988.

GOFERT, L.F. Programas de inseminação artificial em tempo fixo (IATF). Aspectos técnicos e econômicos. In: SIMPÓSIO DE REPRODUÇÃO EM BOVINOS, 1, 2008, Pelotas. Anais... Pelotas: Embrapa Clima Temperado, 2008. p.4147.

MACHADO, R.; BERGAMASCHI, M.A.C.M.; SILVA, J.C.B.; BINELLI, $M$. Estratégias para reduzir a mortalidade embrionária em bovinos: II Protocolo para reduzir a morte embrionária em vacas de leite e em receptoras de embrião. São Carlos. Embrapa Pecuária Sudeste, 2010. 23p. (Boletim de Pesquisa e Desenvolvimento, 27).
MANN, G.E.; LAMMING, G.E. The influence of progesterone during early pregnancy in cattle. Reproduction in Domestic Animals, v.34, p.269-274, 1999.

MENEGHETTI, M.; VASCONCELOS, J.L.M. Mês de parição, condição corporal e resposta ao protocolo de inseminação artificial em tempo fixo em vacas de corte primíparas. Arquivos Brasileira de Medicina Veterinária e Zootecnia, v.60, n.4, p.786-793, 2008.

NEVES, J.P.; MIRANDA, K.L.; TORTORELLA, R.D. Progresso científico em reprodução na primeira década do século XXI. Revista

Brasileira de Zootecnia, v.39, p.414421, 2010.

ROBISON, N.A.; LESLIE, K.E.; WALTON, J.S. Effect of treatment with progesterone on pregnancy rate and plasma concentrations of progesterone in Holstein cow. Journal of Dairy Science, v.72, p.202-207, 1989.

SÁ FILHO, O.G. de; VASCONCELOS, J.L. M. Inseminação artificial em tempo fixo. In: PIRES, A. V. Bovinocultura de corte. Piracicaba: FEALQ, 2010. v.1, p.529-546.

WOOD-FOLLIS, S.L.; KOJIMA, F.N.; LUCY, M.C.; SMITH, M.F.;

PATTERSON , D.J. Estrus synchronization in beef heifers with progestin-based protocols I. Differences in response based on pubertal status at the initiation of treatment.

Theriogenology, v.62, n.8, p.15181528, 2004.

Data de recebimento: 06/11/2013

Data de aprovação: 18/06/2014 